\title{
One-dimensional hard-point gas as a thermoelectric engine
}

\author{
Jiao Wang, ${ }^{1,2}$ Giulio Casati, ${ }^{3,4,5, *}$ Tomaž Prosen, ${ }^{6}$ and C.-H. Lai ${ }^{2,5, \dagger}$ \\ ${ }^{1}$ Temasek Laboratories, National University of Singapore, 117542 Singapore, Singapore \\ ${ }^{2}$ Beijing-Hong Kong-Singapore Joint Center for Nonlinear and Complex Systems (Singapore), National University of Singapore, \\ 117542 Singapore, Singapore \\ ${ }^{3}$ Center for Nonlinear and Complex Systems, Università degli Studi dell'Insubria, Como 22100, Italy \\ ${ }^{4}$ CNR-INFM and Istituto Nazionale di Fisica Nucleare, Sezione di Milano, Milan 20133, Italy \\ ${ }^{5}$ Centre for Quantum Technologies, National University of Singapore, 117543 Singapore, Singapore \\ ${ }^{6}$ Physics Department, Faculty of Mathematics and Physics, University of Ljubljana, Ljubljana 1000, Slovenia \\ (Received 17 February 2009; revised manuscript received 1 June 2009; published 24 September 2009)
}

\begin{abstract}
We demonstrate the possibility to build a thermoelectric engine using a one-dimensional gas of molecules with unequal masses and hard-point interaction. Most importantly, we show that the efficiency of this engine is determined by a parameter $Y T$ which is different from the well known figure of merit $Z T$. Even though the efficiency of this particular model is low, our results shed light on the problem and open the possibility to build efficient thermoelectric engines.
\end{abstract}

DOI: 10.1103/PhysRevE.80.031136

PACS number(s): 05.60.Cd, 84.60.Rb, 05.45.Pq

\section{INTRODUCTION}

Combinations of mathematical results, numerical studies, and even laboratory experiments have greatly improved our understanding of phenomenological transport equations in recent years. The derivation of such equations from purely dynamical laws, classical, or quantum has been one of the main subjects of interest [1]. Even though a complete rigorous picture is still lacking, it is however clear that dynamical chaos is an essential ingredient.

A better understanding of the above problem is important not only from a fundamental point of view in order to provide a justification of phenomenological laws. It is also relevant for several applications. One particular important aspect is the connection with thermoelectric power generation and refrigeration [2-4]. Indeed, due to the increasing environmental concern and energy demand, thermoelectric phenomena are expected to play an increasingly important role in meeting the energy challenge of the future. However, the main difficulty is the poor efficiency of existing devices. Indeed the suitability of a thermoelectric material for energy conversion or electronic refrigeration is characterized by the thermoelectric figure of merit $Z=\sigma S^{2} / \kappa$, where $\sigma$ is the coefficient of electric conductivity, $S$ is the Seebeck coefficient and $\kappa$ is the thermal conductivity. The Seebeck coefficient $S$, also called thermopower, is a measure of the magnitude of an induced thermoelectric voltage in response to a temperature difference.

For a given material and a pair of temperatures $T_{H}$ and $T_{C}$ of hot and cold thermal baths, respectively, $Z$ is related to the efficiency $\eta$ of converting the heat current $J_{Q}$ (between the baths) into the electric power $P$ which is generated by attaching a thermoelectric element to an optimal Ohmic impedance. Namely, in the linear regime (see, e.g., Ref. [2]):

\footnotetext{
*giulio.casati@uninsubria.it

†cqtlch@nus.edu.sg
}

$$
\eta=\frac{P}{J_{Q}}=\eta_{\text {Carnot }} \frac{\sqrt{Z T+1}-1}{\sqrt{Z T+1}+1},
$$

where $\eta_{\text {Carnot }}=1-T_{C} / T_{H}$ is the Carnot efficiency and $T$ $=\left(T_{H}+T_{C}\right) / 2$. Thus a good thermoelectric device is characterized by a large value of the nondimensional figure of merit $Z T$. However, in spite of the fact that the second principle of thermodynamics does not impose any restriction on the value of $Z T$, all attempts to find high $Z T$ values (let us say $Z T$ $>3$ at room temperature) have failed. The problem is that the different transport coefficients $S, \sigma$, and $\kappa$ are interdependent, making the optimization of $Z T$ extremely difficult. We believe that a better understanding of the possible microscopic mechanisms [5] which determine the value of ZT may lead to a substantial improvement.

In a recent paper [6], a dynamical system approach to a Lorenz-gas-type model has been used and an interesting mechanism to reach high $Z T$ value has been discovered. More recently, a one-dimensional diatomic disordered chain of hard-point elastic particles has been considered [7] and it has been found that $Z T$ diverges to infinity with increasing the number of particles inside the chain. This result suggests the possibility to build a thermoelectric engine by connecting two heat baths with two chains of different sizes.

In this paper, we analyze such an engine and we show that, indeed, a nonzero circular current is established inside the system when the stationary state is reached. Quite interestingly the efficiency of such an engine appears to be unrelated to the figure of merit $Z T$ and it remains quite low in spite of the fact that $Z T$ becomes larger and larger with increasing the system size. Indeed we analytically show and numerically confirm that the efficiency of such engine is determined by another figure of merit $Y T$. From one hand the low value of $Y T$ explains the poor efficiency of the engine; on the other hand, it sheds light on future directions for increasing the efficiency of thermoelectric engines.

\section{MODEL OF THE HEAT ENGINE}

We consider a simple model of the engine (see Fig. 1) which consists of two heat baths and two connecting chan- 


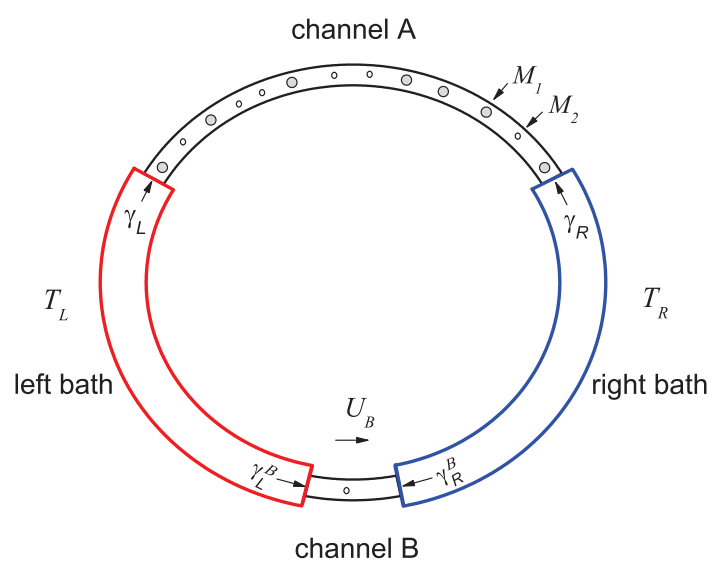

FIG. 1. (Color online) Scheme of the thermoelectric engine studied in this paper. It consists of two heat baths at different temperature $T_{L}$ and $T_{R}$ and two channels of different lengths. See text for the details.

nels $A$ and $B$ of length $L_{A}$ and $L_{B}$, respectively. We assume that the whole system does not exchange particles with the outside. Therefore, the total number of particles $N$ in the two channels and in the two baths remains constant during the simulation. The two heat baths are kept at different temperatures $T_{L}$ and $T_{R}$, and have the same finite length $V$. Each channel is a one-dimensional diatomic disordered chain of hard-point elastic particles with velocities $v_{k}$ and masses $m_{k} \in\left\{M_{1}, M_{2}\right\}$ randomly distributed (we use convenient nondimensional units). The particles interact among each other through elastic collisions only. A collision between two neighboring particles with mass $m_{k}$ and $m_{l}$ causes a change in their velocities $v_{k}$ and $v_{l}$ as $\Delta v_{k}=2 m_{l}\left(v_{l}-v_{k}\right) /\left(m_{k}+m_{l}\right)$ and $\Delta v_{l}=2 m_{k}\left(v_{k}-v_{l}\right) /\left(m_{k}+m_{l}\right)$. An efficient algorithm has been developed which performs correct chronological order of collisions and update particle's positions and velocities in $\sim \log N$ computer operations per collision. We fix the length of the channel $B$ to be small and study how the properties of the engine vary as the length of the channel $A$ is increased. In particular, if channel $B$ is small enough then particles can pass trough it without suffering any collisions.

The heat baths are modeled in the following way. Suppose $n_{1}$ particles of mass $M_{1}$ and $n_{2}$ particles of mass $M_{2}$ are confined in a box of length $V$. The particles collision rate with one end of the box is given by

$$
\gamma=\frac{\rho_{1}}{\rho} \gamma_{1}+\frac{\rho_{2}}{\rho} \gamma_{2}
$$

where $\gamma_{i}=\rho \sqrt{\frac{T}{2 \pi M_{i}}}, \quad \rho_{i}=n_{i} / V, \quad(i=1,2), \rho=n / V$, and $n=n_{1}$ $+n_{2}$. Then the heat bath model is straightforward: if one end of the box is opened, the particles are emitted with the same rate $\gamma$, and the time interval $t$ between two consecutive emissions is a random variable which obeys the distribution

$$
\operatorname{Prob}(t)=\frac{1}{t_{0}} e^{-t / t_{0}}
$$

with $t_{0}=1 / \gamma$. The mass of the emitted particle is assigned to be $M_{i}(i=1,2)$ randomly according to the probability

$$
\Pi_{i}=\frac{\rho_{i} \gamma_{i}}{\rho_{1} \gamma_{1}+\rho_{2} \gamma_{2}}
$$

and its velocity is generated from the distribution

$$
P_{i}(v)=\frac{M_{i}|v|}{T} e^{-M_{i} v^{2} / 2 T}
$$

As expected, when $M_{1}=M_{2}$, the heat bath model of identical particles is recovered. Note that in this paper, nondimensional units are used in which the Boltzmann's constant can be set to unity. Note that the distribution Eq. (5) can be rigorously derived from the Maxwell-Boltzmann distribution, whereas the prefactor $|v|$ simply takes into account the fact that faster particles arrive more often to the point of emission.

The emission rate from the left heat bath into channel $A$ is therefore $\gamma_{L}=\frac{\rho_{1, L}}{\rho_{L}} \gamma_{1, L}+\frac{\rho_{2, L}}{\rho_{L}} \gamma_{2, L}$ where $\rho_{L}$ is the total particle number density at the left heat bath, $\rho_{i, L}$ is that of particles with mass $M_{i}$, and $\gamma_{i, L}=\rho_{L} \sqrt{\frac{T_{L}}{2 \pi M_{i}}}(i=1,2)$. Similarly one can write the expression for the emission rate $\gamma_{R}$ from the right heat bath.

As to the channel $B$ we assume that the emission rates are proportional to $\gamma_{L}$ and $\gamma_{R}$ :

$$
\gamma_{L}^{B}=r \gamma_{L}, \quad \gamma_{R}^{B}=r \gamma_{R},
$$

where $r$ is an adjustable parameter. For $r=0$ channel $B$ is in fact closed hence there is no net particle current.

Conversely, whenever a particle from each channel arrives at the border with the bath it is absorbed by the bath, so that a stationary state is established after sufficiently long time. If the stationary state is such that there is a net particle current around the system, then one can use it to extract work. To this end we insert inside the channel $B$ an auxiliary potential $U_{B}$ which the particles have to climb thus performing useful work. Let us first consider the case where the channel $B$ is short enough (i.e., $L_{B}=1$ ) so that the probability of particles collision inside it is negligible. Suppose $T_{L}$ $>T_{R}$ then the current runs clockwise at $U_{B}=0$ due to the pressure balance between the two heat baths. Thus a particle emitted from the right heat bath can pass through the channel $B$ only when its kinetic energy is larger than $U_{B}$, otherwise it will return back. Therefore the probability with which the emitted particle passes through the channel $B$ is

$$
\mathcal{P}_{R L}=e^{-U_{B} / T_{R}}
$$

for both particles of masses $M_{1}$ and $M_{2}$. Hence the work extracted per unit of time is

$$
P=r\left(\gamma_{R} \mathcal{P}_{R L}-\gamma_{L}\right) U_{B}
$$

The efficiency of the engine thus reads

$$
\eta=\frac{P}{J_{Q, A}+J_{Q, B}}
$$

where $J_{Q}^{A}$, and $J_{Q}^{B}$, are the net thermal energy flows from the left heat bath in a unit time into the channel $A$ and $B$, respectively, which can be measured by numerical simulations. No- 


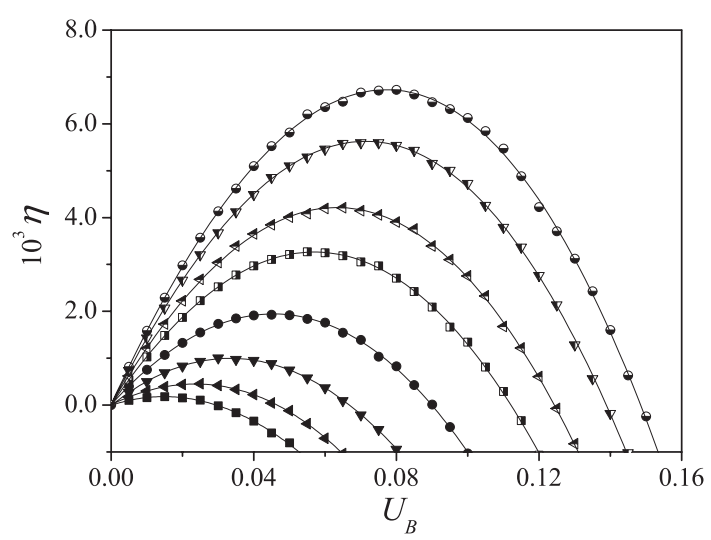

FIG. 2. Efficiency of the engine model illustrated in Fig. 1 as a function of the potential $U_{B}$ for $L_{A}=4,8,16,32,64,100,200$, and 400 (from below to up). $T_{L}=1.2, T_{r}=0.8$, and $r=1$. The length of channel $B$ is unity.

tice that, in particular, $J_{Q}^{B}$ can be expressed analytically through

$$
J_{Q, B}=r\left(T_{L} \gamma_{L}-\mathcal{P}_{R L} T_{R} \gamma_{R}\right)
$$

As it is seen from Fig. 2 our engine works. Here we plot the numerically computed efficiency $\eta$ against the potential $U_{B}$ for various lengths of the channel $A$. The solid curves are polynomial (three-order) fittings of the numerical data.

In this calculation the length of channel $B$ is set to unity and the length of both heat baths is very large, namely, $V$ $=10^{3}$. The total number of particles in the system is set to be $N=2 V+L_{A}+L_{B}$ such that the overall averaged particle density is unity. The two types of molecules are set to be equal in number and have masses $M_{1}=1$ and $M_{2} \approx 0.618$, respectively. In addition, for a given length $L_{A}$ of the channel $A$, there is an optimal value of $U_{B}$ such that the efficiency is maximized. Denoting the maximum efficiency by $\eta_{\max }$, it is interesting to compute the dependence of $\eta_{\max }$ on $L_{A}$ and compare it with theoretical expectations.

Extensive and accurate numerical computations, summarized in Fig. 3, show that even though $\eta_{\max }$ increases with $L_{A}$, i.e., with the average number of molecules in the channel $A$, the increasing rate slows down very fast. Also it seems hard to find a simple fitting for the dependence of $\eta_{\max }$ on $L_{A}$.

We should notice that $\eta_{\max }$ depends also on $T_{L}$ and $T_{R}$. However if $\eta_{\max }$ is rescaled to the Carnot efficiency, its behavior with $L_{A}$ is the same for all $\left(T_{L}, T_{R}\right)$, pairs, given that the temperature difference is small enough. This can be seen clearly in Fig. 3.

Remarks: we have also investigated carefully several variants of the engine model. First, we have studied the dependence of $\eta_{\max }$ on $r$. We have found that indeed $\eta_{\max }$ can be slightly improved by adjusting $r$ (e.g., $\sim 30 \%$ as compared to Fig. 3), however the efficiency remains very low. Then we have checked whether adding a potential $U_{A}$ against the current in the channel $A$ can improve efficiency. In this case a particle in the channel $A$ would undergo a parabolic motion between two consecutive collisions with its neighbors. This

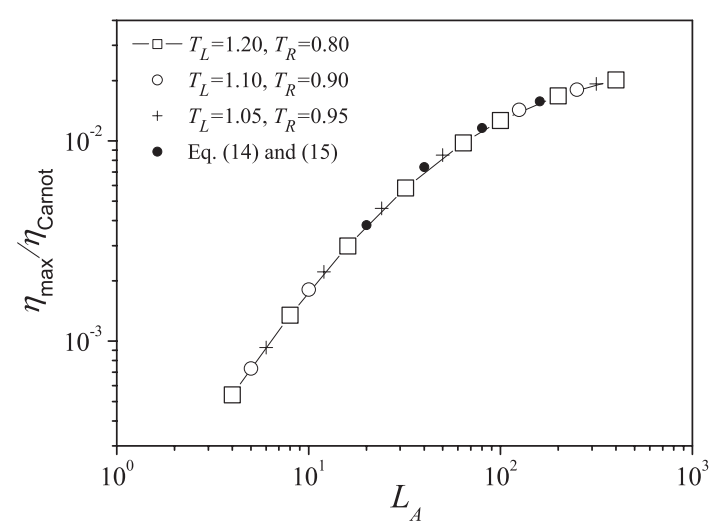

FIG. 3. The maximum efficiency of the engine for different temperature gradients. Here $r=1$ and $L_{B}=1$. The solid dots are obtained from Eqs. (14) and (15), where the parameters $\kappa, \sigma$, and $S$ for both channels are numerically obtained as in Fig. 4. As it is seen there is perfect agreement with the directly numerically computed efficiency.

variant does not increase the maximum efficiency $\eta_{\max }$ either which has been found to depend only on the sum $U_{A}+U_{B}$ rather than on $U_{A}$ and $U_{B}$ separately. Also a longer length of channel $B$ has been considered but it turns out that for a given $L_{A}+L_{B}$, the efficiency reaches its highest value when $L_{B} \leq 1$. To summarize, the various modifications to the engine model we have considered do not improve the efficiency significantly.

\section{ENGINE'S FIGURE OF MERIT}

Let us now explain why our engine works at a rather low efficiency in spite of the fact that $Z T$ goes to infinity. In order to derive a theoretical expression for the efficiency we use thermodynamic linear response relations for the heat currents $J_{Q, A}, J_{Q, B}$, and for the particle currents $J_{\rho, A}, J_{\rho, B}$, in the channels $A$ and $B$, respectively, namely,

$$
\begin{gathered}
J_{Q, A}=-\kappa_{A}^{\prime} \Delta T / L_{A}-T \sigma_{A} S_{A}\left(\Delta \mu / L_{A}+U_{A}\right) \\
J_{\rho, A}=-\sigma_{A} S_{A} \Delta T / L_{A}-\sigma_{A}\left(\Delta \mu / L_{A}+U_{A}\right) \\
J_{Q, B}=-\kappa_{B}^{\prime} \Delta T / L_{B}-T \sigma_{B} S_{B}\left(\Delta \mu / L_{B}-U_{B}\right) \\
J_{\rho, B}=-\sigma_{B} S_{B} \Delta T / L_{B}-\sigma_{B}\left(\Delta \mu / L_{B}-U_{B}\right)
\end{gathered}
$$

where $\kappa_{A}^{\prime}=\kappa_{A}+T \sigma_{A} S_{A}^{2}, \kappa_{B}^{\prime}=\kappa_{B}+T \sigma_{B} S_{B}^{2}, \mu$ is the chemical potential, $\Delta T=T_{R}-T_{L}$, and $\Delta \mu=\mu_{R}-\mu_{L}$. In addition, in the stationary state we must also have

$$
J_{\rho, A}+J_{\rho, B}=0 .
$$

The relations given by Eqs. (11) and (12) represent five linear equations in the five unknowns $J_{Q, A}, J_{Q, B}, J_{\rho, A}, J_{\rho, B}$, and $\Delta \mu$, whereas the other parameters, such as transport or Onsager coefficients are considered to be known. From the solutions of the above equations we compute the efficiency $\eta$ as a function of the two bias potentials: 


$$
\eta\left(U_{A}, U_{B}\right)=\frac{P}{J_{Q}}=\frac{J_{\rho, A} U_{A}-J_{\rho, B} U_{B}}{J_{Q, A}+J_{Q, B}} .
$$

Since $\eta\left(U_{A}, U_{B}\right)$ is a quadratic function, we can easily maximize it by computing the optimal values of $U_{A}, U_{B}$. The final result can be expressed in a simple form as

$$
\eta_{\max } / \eta_{\text {Carnot }}=1-2 \sqrt{(Y T)^{-2}+(Y T)^{-1}}+2(Y T)^{-1}
$$

with a figure of merit which we introduce as

$$
Y T=\frac{\left(\sigma_{A} / L_{A}\right)\left(\sigma_{B} / L_{B}\right)}{\left(\sigma_{A} / L_{A}+\sigma_{B} / L_{B}\right)} \frac{\left(S_{A}-S_{B}\right)^{2}}{\left(\kappa_{A} / L_{A}+\kappa_{B} / L_{B}\right)} T
$$

From Eq. (14) it can be seen that efficiency is given by this figure of merit exclusively and it has nothing to do with the merit $\quad Z T$. Clearly, as $Y T \rightarrow 0(Y T \rightarrow \infty)$ we have $\eta_{\max } \rightarrow 0\left(\eta_{\max } \rightarrow \eta_{\text {Carnot }}\right)$.

In order to understand why our engine model has a low efficiency, we investigate carefully the dependence of the parameters $\sigma, \kappa$, and $S$ on the channel length. These quantities can be obtained from the Onsager coefficients $L_{u u}, L_{u \rho}, L_{\rho u}, L_{\rho \rho}[6]$ which in turn are calculated by measuring the particles current $J_{\rho}$, and the energy current $J_{u}$, when the stationary state of the system is reached through

$$
\begin{aligned}
& J_{u}=L_{u u} \Delta \beta / L-L_{u \rho} \Delta \alpha / L \\
& J_{\rho}=L_{\rho u} \Delta \beta / L-L_{\rho \rho} \Delta \alpha / L
\end{aligned}
$$

with $\alpha \equiv \mu / T, \beta \equiv 1 / T$ and $\mu$ the chemical potential:

$$
\mu / T=\left(\rho_{1} \ln \rho_{1}+\rho_{2} \ln \rho_{2}\right) / \rho-\ln \sqrt{T}+\text { const. }
$$

In Fig. 4 we present the numerical results for a onedimensional (1D) mixed gas chain with $M_{1}=1, M_{2} \approx 0.618$, $T=1$, and $2 \rho_{1}=2 \rho_{2}=\rho=1$. The constant in Eq. (17) is set to be $\ln 2+1$ such that $\mu=1$. The results clearly show that, as the channel length is increased, while the ratio $\sigma / \kappa$ increases as a power law, the Seebeck coefficient $S$ undergoes a slower and slower increase. As a result, while $Z T$ increases indefinitely with the channel length $L$, the merit $Y T$ remains small.

\section{CONCLUSIONS}

In summary, for the engine model (Fig. 1) based on a one-dimensional mixed gas, the efficiency is governed by a
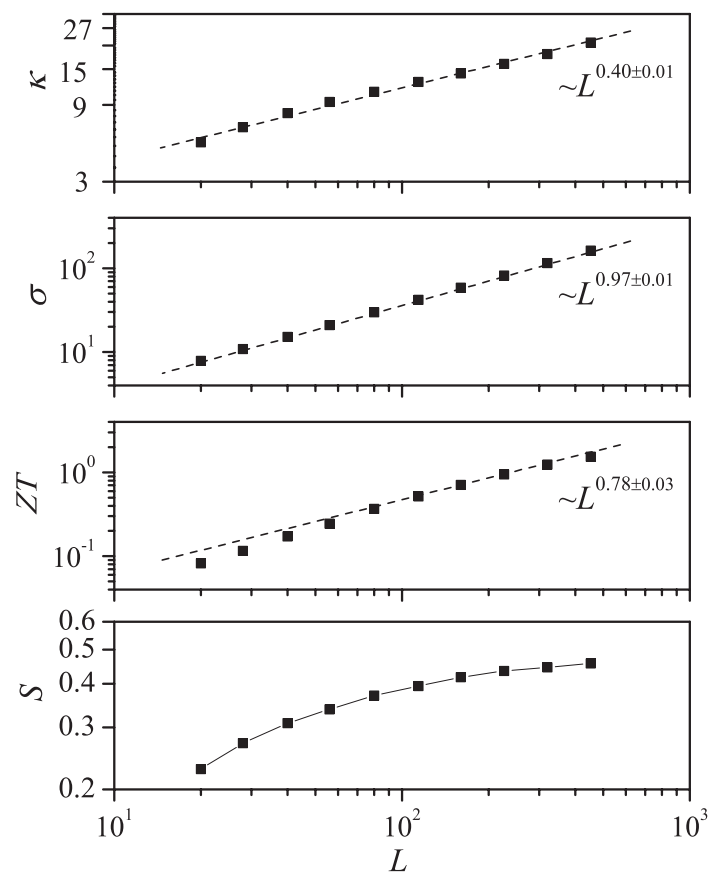

FIG. 4. Dependence on the channel length of various parameters of the 1D mixed gas. From these results it is clear why when $Z T$ can be very large for a long channel, the merit $Y T$ that governs the efficiency of the engine model (Fig. 1) takes a lower value instead.

figure of merit introduced in this article. Based on its relation to the thermoelectric parameters [Eq. (15)], it can be expected that an efficient mixed gas type engine should be such that its Seebeck coefficient changes fast when the channel length is changed. Such a mixed gas might be that consisting of three or more types of molecules. We believe that our method of analysis of thermoelectric or thermochemical heat engines should be applicable to a wide range of models which consist of two transport channels between a pair of baths.

\section{ACKNOWLEDGMENTS}

J.W. acknowledges support from the Defense Science and Technology Agency (DSTA) of Singapore under agreement of No. POD0613356 and T.P. acknowledges Grants No. P10044 and No. J1-7347 of the Slovenian research agency.
[1] S. Lepri, R. Livi, and A. Politi, Phys. Rep. 377, 1 (2003); A. Dhar, Adv. Phys. 57, 457 (2008); F. Bonetto, J. L. Lebowitz, and L. Ray Bellet, in Mathematical Physics 2000, edited by A. Fokas, A. Grigoryan, T. Kibble, and B. Zegarlinski (Imperial College Press, London, 2000) pp. 128-150; G. Casati, J. Ford, F. Vivaldi, and W. M. Visscher, Phys. Rev. Lett. 52, 1861 (1984).

[2] G. Mahan, B. Sales, and J. Sharp, Phys. Today 50 (3), 42 (1997).
[3] A. Majumdar, Science 303, 777 (2004).

[4] M. S. Dresselhaus et al., Adv. Mater. 19, 1043 (2007).

[5] T. E. Humphrey and H. Linke, Phys. Rev. Lett. 94, 096601 (2005).

[6] G. Casati, C. Mejia-Monasterio, and T. Prosen, Phys. Rev. Lett. 101, 016601 (2008).

[7] G. Casati, L. Wang, and T. Prosen, J. Stat. Mech. (2009) L03004. 